\title{
Evaluación radiográfica de la posición del túnel femoral y tibial en reconstrucción de ligamento cruzado anterior con técnica anatómica
}

\author{
Radiographic evaluation of femoral and tibial tunnel position in anterior \\ cruciate ligament reconstruction with anatomic technique \\ García-Dobarganes-Barlow FE, ${ }^{*}$ Uribe-Chávez JM,${ }^{\ddagger}$ García-Munguía FA, ${ }^{\S}$ \\ Guevara-Álvarez A, ${ }^{\S}$ Moreno-Carranza B, ${ }^{\circledR}$ Negrete-Corona J, ${ }^{\S}$ Archila-López OE ${ }^{\ddagger}$ \\ Hospital Ángeles de Querétaro, Querétaro, Qro.
}

\begin{abstract}
RESUMEN. Introducción: En la reconstrucción de ligamento cruzado anterior se intenta reproducir ligamento nativo, realizar túneles independientes, optimizar los puntos de entrada y la efectividad de la anatomía; hacer mediciones radiográficas de rodilla, identificar la posición del túnel femoral y tibial en pacientes operados de reconstrucción de ligamento cruzado anterior con técnica anatómica. Material y métodos: Se identificaron pacientes en el período de Enero de 2018 a Diciembre de 2019. Se evaluaron de manera retrospectiva 98 pacientes. Mediciones radiográficas en fémur: se determinó el ángulo de inclinación del túnel, el sitio de inserción con respecto a la línea de Blumensaat y la distancia transósea. En tibia: la ubicación en porcentaje de la localización de platillo tibial en proyección anteroposterior y lateral, ángulo de túnel tibial. Resultados: Los pacientes postoperados fueron 98, masculinos $(75.5 \%)$ y femeninos $(24.5 \%)$. Postoperados en el año 2018 (57.15\%) y en 2019 (42.85\%). Rango de edad: el grupo afectado fue de 21-25 años, lado afectado: derecho en $57.15 \%$. Mediciones radiográficas en fémur: el ángulo de
\end{abstract}

ABSTRACT. Introduction: Reconstruction of the anterior cruciate ligament attempts to reproduce the native ligament; make independent tunnels optimize the entry points and effectiveness the anatomy. Perform radiographic measurements of the knee, identify position of femoral and tibial tunnels in patients operated for reconstruction of anterior cruciate ligament with anatomical technique. Material and methods: Patients were identified in the period January 2018 to December 2019. 98 patients were retrospectively evaluated. Radiographic measurements in the femur, the inclination angle of the tunnel, the insertion site with respect to the Blumensaat line and the transosseous distance were determined. In tibia, the location in percentage of the tibial plateau location in anteroposterior and lateral view, tibial tunnel angle. Results: Postoperative patients were 98. (75.5\%) male, female (24.5\%). Postoperative in 2018 (57.15\%) and 2019 (42.85\%) Age range: affected group was $21-25$ years, right side affected in $57.15 \%$; Radiographic measurements in femur, the angle of inclination of the tunnel

\section{Nivel de evidencia: IV}

\footnotetext{
* Jefe del Curso de Alta Especialidad en Cirugía Articular. Instituto Queretano de Alta Especialidad en Ortopedia (IQAEO). Hospital Ángeles de Querétaro, Querétaro, Qro.

${ }^{*}$ Fellow del Curso de Alta Especialidad en Cirugía Articular. Instituto Queretano de Alta Especialidad en Ortopedia (IQAEO). Hospital Ángeles de Querétaro. Querétaro, Qro.

${ }^{\S}$ Profesor adjunto del Curso de Alta Especialidad en Cirugía Articular. Instituto Queretano de Alta Especialidad en Ortopedia (IQAEO). Hospital Ángeles de Querétaro. Querétaro, Qro.

^ Coordinadora de Investigación. Escuela de Ciencias de la Salud. Universidad Anáhuac, Querétaro.

Correspondencia:

Fidel Enrique García-Dobarganes-Barlow

E-mail: fdobarganes@icloud.com
}

Recibido: 09-02-2021. Aceptado: 20-11-2021.

Citar como: García-Dobarganes-Barlow FE, Uribe-Chávez JM, García-Munguía FA, Guevara-Álvarez A, Moreno-Carranza B, NegreteCorona J, et al. Evaluación radiográfica de la posición del túnel femoral y tibial en reconstrucción de ligamento cruzado anterior con técnica anatómica. Acta Ortop Mex. 2021; 35(4): 327-330. https://dx.doi.org/10.35366/103312

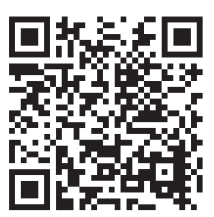


inclinación del túnel fue de $45^{\circ}$, el porcentaje en la línea de Blumensaat fue de $20 \%$ y la distancia transósea fue $3.43 \mathrm{~cm}$. En tibia la distancia porcentual en proyección anteroposterior fue de $44 \%$ y lateral de $28 \%$. El ángulo en túnel tibial en proyección anteroposterior fue de $73^{\circ}$ y lateral de $114^{\circ}$. Conclusión: La técnica anatómica es una buena opción para efectuar túneles con posición anatómica óptima. Realizar túneles independientes permite mejorar la cobertura de la huella anatómica normal, aumenta los grados y la inclinación de túneles y provoca lesiones agregadas.

Palabras clave: Ligamento cruzado, reconstrucción, túneles, ángulo.

\section{Introducción}

La posición del injerto en la reconstrucción del ligamento cruzado anterior (LCA) y su similitud con la anatomía normal es uno de los principales factores a tener en cuenta para el éxito de esta cirugía. Si revisamos la bibliografía de los últimos años con respecto a las plásticas artroscópicas del LCA, podemos observar una clara tendencia técnica a realizar maniobras y estrategias quirúrgicas que intentan llegar a un concepto de reconstrucción anatómica, colocando túneles femorales y tibiales lo más cerca posible a la huella de inserción del ligamento cruzado anterior normal.

La gran mayoría de las técnicas actualmente utilizadas, denominadas anatómicas, ${ }^{2,3}$ parten de la premisa de que para poder controlar mejor la ubicación del túnel femoral y tibial, éstas deben hacerse en forma independiente y este concepto técnico permitirá actuar sobre dos factores importantes en la reproducción anatómica de la huella femoral: el sitio de entrada y la oblicuidad del túnel.

El objetivo del presente trabajo es evaluar y realizar mediciones radiográficas en proyecciones anteroposterior y lateral de rodilla para identificar la posición del túnel femoral y tibial en pacientes operados de reconstrucción de ligamento cruzado anterior con técnica anatómica.

\section{Material y métodos}

Entre Enero de 2018 y Diciembre de 2019 se evaluaron radiológicamente 98 pacientes con plástica de ligamento cruzado anterior primaria en forma retrosprospectiva. Los criterios de inclusión fueron: edad de entre 16 y 56 años y reconstrucción primaria de ligamento cruzado anterior con autoinjerto y aloinjerto. Los criterios de exclusión fueron cirugías previas en la rodilla a intervenir.

Se les tomó radiografías de frente y perfil en formato digital, utilizando luego el programa de visualización DICOM ${ }^{\circledR}$ Horos versión 5.8.1 para realizar las mediciones.

Todos los pacientes fueron operados por el mismo cirujano con técnica anatómica. Se identifica la localización del túnel femoral. was $45^{\circ}$, percentage in the Blumensaat line was $20 \%$, and the transosseous distance was $3.43 \mathrm{~cm}$. In tibia the percentage distance in anteroposterior projection was $44 \%$, and lateral $28 \%$, The angle in tibial tunnel anteroposterior projection of 73 degrees, and lateral 114. Conclusion: The anatomical technique is a good option to perform tunnels with an optimal anatomical position. By making independent tunnels it allows to improve coverage of normal antomic footprint. Increases degrees and inclination of tunnels, causes added injuries.

Keywords: Cruciate ligament, reconstruction, tunnels, angle.

Se utilizó la misma fijación tibial en ambos grupos, un tornillo bioabsorbible y botón cortical (Ultrabutton) ${ }^{\circledR}$.

Radiográficamente evaluamos en el fémur: el ángulo de inclinación del túnel femoral, tomando una línea recta que pasa por el centro de la diáfisis femoral y otra línea que pasa por el centro del túnel femoral, ${ }^{4}$ el sitio de inserción con respecto a la línea de Blumensaat en forma porcentual, ${ }^{5}$ tomando la longitud de la línea de Blumensaat en $\mathrm{cm}$ y luego midiendo de la cortical posterior la distancia en $\mathrm{cm}$ al centro del túnel femoral y la distancia transósea del fémur medida desde el intercóndilo hasta la cortical femoral a la altura del botón femoral o proyectando una línea a través del centro del túnel femoral hasta la cortical femoral (Figura 1).

Para evaluar la ubicación del túnel tibial ${ }^{6}$ se midió la ubicación en porcentaje de la localización con respecto al platillo tibial en el frente, se midió el ancho del platillo tibial y la distancia entre el extremo medial del platillo y el centro del túnel tibial expresado en forma porcentual. En el perfil se midió la distancia anteroposterior del platillo tibial interno y la distancia de la cara anterior de la tibia al centro del túnel tibial expresado en forma porcentual. El ángulo del túnel tibial en el frente es evaluado por una línea que pasa por el platillo tibial interno y su intersección con una línea que pasa por el centro del túnel tibial. El ángulo de inclinación del túnel tibial en el perfil es medido por una línea que pasa por el platillo tibial interno de anteroposterior y otra línea que pasa por el centro del túnel tibial que lo intercepta (Figura 2).

\section{Resultados}

De los 98 pacientes registrados, $75.5 \%$ fueron masculinos y $24.5 \%$ femeninos. Postoperados en 2018 (57.15\%) y en 2019 (42.85\%). Se presentó una edad media de 30 años con una moda de 25 años y mediana de 26 años.

Las mediciones radiográficas en fémur: el ángulo de inclinación del túnel fue de $45^{\circ}$, el porcentaje en la línea de Blumensaat de $20 \%$ y la distancia transósea fue de $3.43 \mathrm{~mm}$.

En tibia, la distancia porcentual en proyección anteroposterior fue de $44 \%$ y en la lateral de $28 \%$; el ángulo del 
túnel tibial en proyección anteroposterior fue de $73^{\circ} \mathrm{y}$ en la proyección lateral fue de $114^{\circ}$.

\section{Discusión}

El uso de imágenes radiológicas en dos planos es un método de evaluación postoperatorio de rutina, sencillo y de bajo costo, que permite determinar la posición del túnel tibial y femoral cuando llevamos a cabo reconstrucciones artroscópicas del LCA. Con el uso de técnicas transportales es posible realizar túneles tibiales y femorales en posiciones que imiten la anatomía normal, pero en términos de cobertura de huellas no lograrían el mismo resultado. Altcheck y colaboradores ${ }^{3}$ determinaron mediante estudios de resonancia de alta definición y una posterior reconstrucción $3 \mathrm{D}$ que se podía colocar en túnel femoral cubriendo óptimamente la huella femoral utilizando la técnica anatómica, pero también detectaron que para lograr esa cobertura, debía hacerse a expensas de la ubicación del túnel tibial, observándose una cobertura de la huella de $66.3 \%$ como media (rango entre 100-44.6\%). Con la técnica transportal mediante estudios anatómicos habían llegado a la misma conclusión, asegurando que para lograr un túnel femoral que cubra completamente la huella, el túnel tibial debe iniciarse muy cerca de la interlínea articular y muy horizontal generando como consecuencia un túnel más corto.

Algunos autores relacionan la ubicación de los túneles con el resultado clínico y aconsejan que la ubicación del túnel tibial sea en el sector posteromedial de la huella y no en el centro para evitar de esta manera los síndromes de fricción del injerto en el techo del intercónilo. ${ }^{7,8,9}$ Moisala y colaboradores ${ }^{10}$ luego de avaluar 102 pacientes, determinaron que los mejores resultados se obtuvieron cuando el injerto estaba lo más posterior posible en el fémur y lo más anterior posible en la tibia; ubican el túnel femoral entre 25$29 \%$ de la línea de Blumensaat. Nuestra investigación radiológica demostró túneles femorales de ambos grupos entre $12-41 \%$, siendo los del grupo TP un poco más anteriores y aparentemente sin repercusión clínica al momento de cerrar el estudio.

En cuanto a la posición del túnel tibial, estos mismos autores la ubican entre $32-37 \%$ de la cara anterior de la tibia.
En nuestro grupo se ubicaron un poco más anteriores sin que ello haya producido clínicamente una limitación de extensión de la rodilla al momento de cerrar el estudio.

Pinczewski, ${ }^{11}$ luego de evaluar de manera prospectiva a 200 pacientes, mostró que los resultados óptimos después de una reconstrucción de ligamento cruzado anterior con isquiotibiales dependían de la orientación de los túneles. Observó un elevado índice de rerruptura si no se ubicaba el túnel femoral en $86 \%$ posterior de la línea de Blumensaat, medición que nosotros omitimos por no tener una radiografía lateral en extensión. El túnel tibial en el perfil se ubicaría en $48 \%$ o menos, ya que en más de $50 \%$ se observó un elevado índice de rerruptura.

El ángulo de inclinación femoral fue de $19^{\circ} \mathrm{o}$ menos en los pacientes con pivot grado 1 o menor. Según estos trabajos, la orientación del túnel y del injerto sería vital para obtener propiedades biomecánicas casi normales y una buena funcionalidad de la reconstrucción. Las mediciones radiográficas obtenidas en el presente trabajo son consistentes con los valores publicados como óptimos de una reconstrucción anatómica del LCA. ${ }^{12}$

Estudios anatómicos recientes ${ }^{13,14}$ mostraron que se obtenían túneles con mejor cobertura de la huella y con mejor inclinación del injerto cuando éstos se realizaban utilizando el criterio de independencia. Las fortalezas de este trabajo radican en que todos los casos fueron operados por el mismo grupo quirúrgico, empleando la misma técnica quirúrgica e implantes y que en todos se utilizaron criterios de reconstrucción anatómicos. Las debilidades son la falta de una proyección lateral de la rodilla en extensión, el no hacer una correlación clínica pertinente con la ubicación de los túneles y la no utilización de imágenes de evaluación en forma tridimensional.

\section{Conclusión}

El túnel femoral y los ángulos de inclinación se pueden usar como medidas rápidas y simples en radiografías de PA con soporte de pesas de flexión de $45^{\circ}$ y de manera retrospectiva. Es probable que un ángulo del túnel femoral de menos de $32.7^{\circ}$ y un ángulo de inclinación mayor de $55^{\circ}$ tengan reconstrucciones de LCA insuficientes y con mayor riesgo
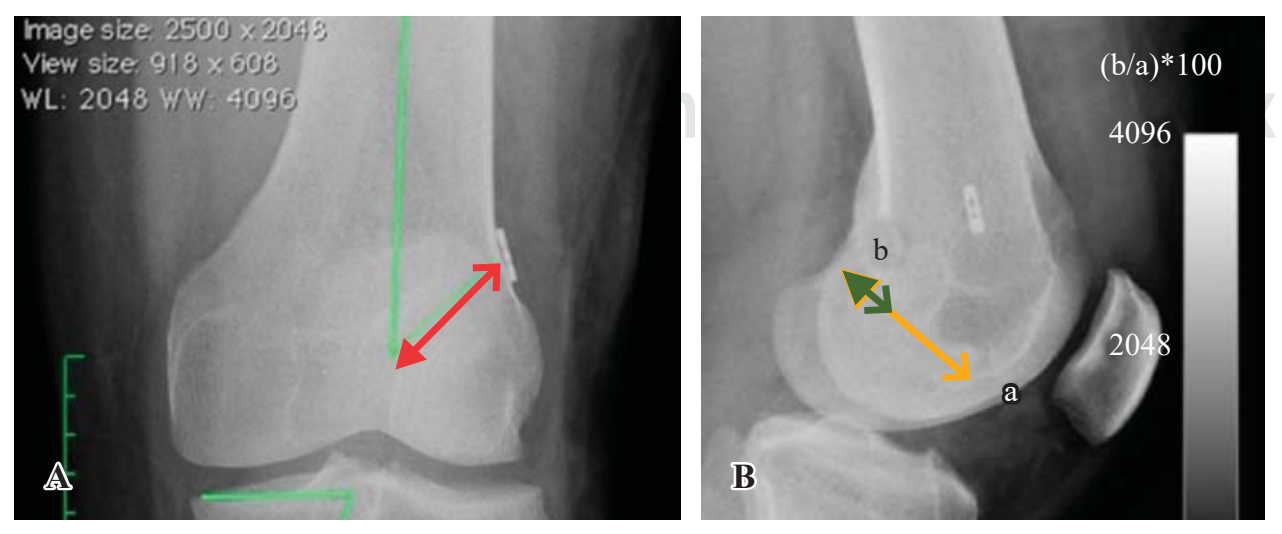

Figura 1:

A) Ángulo de inclinación femoral (líneas verdes) y la distancia transósea (línea roja con dos flechas). B) Se marca la línea de Blumensaat (a) y la distancia del túnel femoral a la pared posterior (b). 


\section{Figura 2:}

A) Distancia mediolateral del platillo tibial (a) y la distancia del túnel tibial a la cortical medial

(b) y la medición porcentual. La línea verde evidencia el ángulo del túnel tibial. B) Distancia posteroanterior del platillo tibial interno (a) y la distancia del túnel tibial a la cortical anterior (b). El ángulo del túnel tibial con respecto al platillo tibial (línea verde)
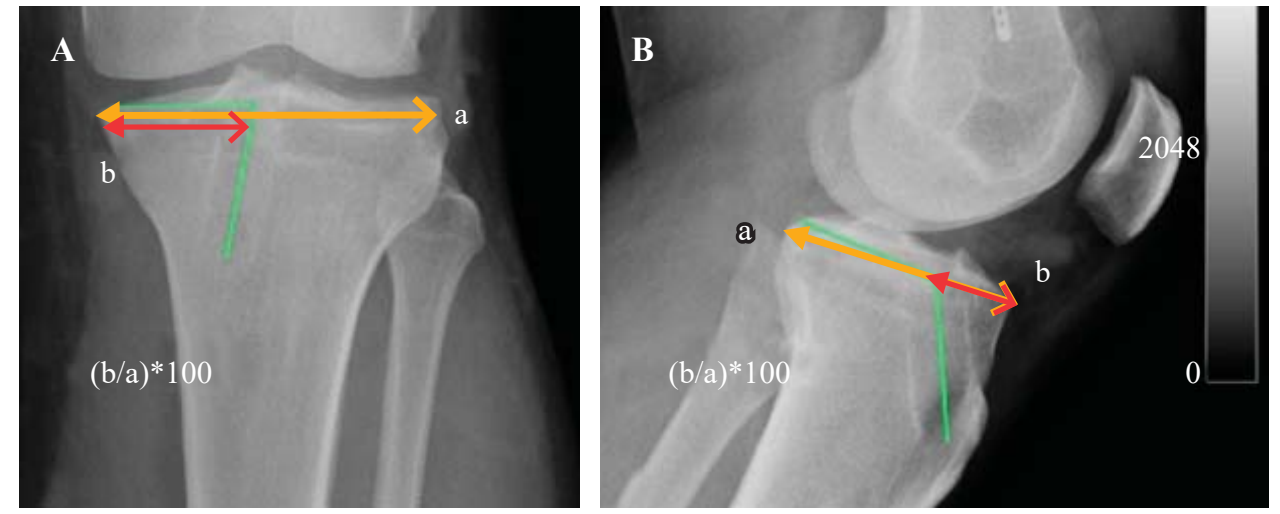

de desarrollar lesiones agregadas (ruptura de injerto, lesiones meniscales laterales, lesiones condrales).

Nuestros resultados muestran que el centro anatómico de la huella femoral del LCA es $43 \%$ de la longitud proximal a distal de la pared de la muesca intercondilar femoral lateral y a $2.5 \mathrm{~mm}$ anterior al margen articular posterior.

\section{Referencias}

1. Jepsen CF, Lundberg-Jensen AK, Faunoe P. Does the position of the femoral tunnel affect the laxity or clinical outcome of the anterior cruciate ligament-reconstructed knee? A clinical, prospective, randomized, double-blind study. Arthroscopy. 2007; 23(12): 1326-33.

2. Abebe ES, Moorman CT 3rd, Dziedzic TS, Spritzer CE, Cothran RL, Taylor DC, et al. Femoral tunnel placement during anterior cruciate ligament reconstruction: an in vivo imaging analysis comparing transtibial and 2-incision tibial tunnel-independent techniques. Am J Sports Med. 2009; 37(10): 1904-11.

3. Bedi A, Altchek DW. The "footprint" anterior cruciate ligament technique: an anatomic approach to anterior cruciate ligament reconstruction. Arthroscopy. 2009; 25(10): 1128-38.

4. Illingworth KD, Hensler D, Working ZM, Macalena JA, Tashman $\mathrm{S}$, Fu FH. A simple evaluation of anterior cruciate ligament femoral tunnel position: the inclination angle and femoral tunnel angle. Am J Sports Med. 2011; 39(12): 2611-18.

5. Piefer JW, Pflugner TR, Hwang MD, Lubowitz JH. Anterior cruciate ligament femoral footprint anatomy: systematic review of the $21 \mathrm{st}$ century literature. Arthroscopy. 2012; 28(6): 872-81.

6. Hwang MD, Piefer JW, Lubowitz JH. Anterior cruciate ligament tibial footprint anatomy: systematic review of the 21 st century literature. Arthroscopy. 2012; 28(5): 728-34.
7. Lee MC, Seong SC, Lee S, Chang CB, Park YK, Jo H, et al. Vertical femoral tunnel placement results in rotational knee laxity after anterior cruciate ligament reconstruction. Arthroscopy. 2007; 23(7): 771-8.

8. Scopp JM, Jasper LE, Belkoff SM, Moorman CT 3rd. The effect of oblique femoral tunnel placement on rotational constraint of the knee reconstructed using patellar tendon autografts. Arthroscopy. 2004; 20(3): 294-9.

9. Yamamoto Y, Hsu WH, Woo SL, Van Scyoc AH, Takakura Y, Debski RE. Knee stability and graft function after anterior cruciate ligament reconstruction: a comparison of a lateral and an anatomical femoral tunnel placement. Am J Sports Med. 2004; 32(8): 1825-32.

10. Moisala AS, Jarvela T, Harilainen A, Sandelin J, Kannus P, Jarvinen $M$. The effect of graft placement on the clinical outcome of the anterior cruciate ligament reconstruction: a prospective study. Knee Surg Sports Traumatol Arthrosc. 2007; 15(7): 879-87.

11. Pinczewski LA, Salmon LJ, Jackson WMF, von Bormann RPB, Haslam PG, Tashiro S. Radiological landmarks for placement of the tunnels in single-bundle reconstruction of the anterior cruciate ligament. J Bone Joint Surg Br. 2008; 90(2): 172-9.

12. Gougoulias N, Khanna A, Griffiths D, Maffulli N. ACL reconstruction: can the transtibial technique achieve optimal tunnel positioning? A radiographic study. Knee. 2008; 15(6): 486-90. doi: 10.1016/j. knee.2008.07.006.

13. Heming JF, Rand J, Steiner ME. Anatomical limitations of transtibial drilling in anterior cruciate ligament reconstruction. Am J Sports Med. 2007; 35(10): 1708-15

14. Rue JP, Ghodadra N, Bach BR Jr. Femoral tunnel placement in single-bundle anterior cruciate ligament reconstruction. A cadaveric study relating transtibial lateralized femoral tunnel position to the anteromedial and posterolateral bundle femoral origins of the anterior cruciate ligament. Am J Sports Med. 2008; 36: 73-9. 